\title{
Influence of palatoplasty on occlusion of patients with isolated cleft palate
}

Omar Gabriel da Silva Filho', Elisa Teixeira Marinho², Rita de Cássia Moura Carvalho Lauris ${ }^{3}$

Objective: This study compared the dental arch morphology of adult patients with isolated cleft palate in order to verify the influence of palatoplasty on occlusion.

Methods: Cast models of 77 patients, 30 males and 47 females, with an average age of 21 years and no syndromes were taken. They were in the permanent dentition and had not undergone orthodontic treatment. The sample was divided into non-operated and operated patients, the latter having been submitted to palatoplasty at a mean age of 2.2 years.

Results: Almost $80 \%$ of the sample exhibited sagittal discrepancies in the inter-arch relationship, with a Class II malocclusion prevailing (59.74\%) followed by Class III (20,78\%), regardless of palatoplasty. Transverse analysis showed a $23 \%$ incidence of posterior crossbite also not influenced by palatoplasty. Intra-arch relationship indicated that constriction and crowding on the upper arch were more frequent in the operated group ( $\mathrm{p}=0.0238$ and $\mathrm{p}=0.0002$, respectively), showing an influence of palatoplasty on its morphology. The predominant morphological characteristics in patients with isolated cleft palate were a Class II malocclusion, upper dental arch constriction and upper and lower anterior crowding.

Conclusion: The influence of palatoplasty was restricted to constriction and crowding of the upper dental arch, with no interference from the extension of the cleft, except for the upper crowding, which occurred more in patients with complete cleft palates.

Keywords: Dental arch. Cleft palate. Dental occlusion.

${ }^{1}$ Orthodontist of the dental division of the Craniofacial Anomalies Rehabilitation Hospital, (HRAC/USP), Brazil. Director of the Course in Preventive and Interceptive Orthodontics of the Society for Social Inclusion of the Cleft Palate (PROFIS), Brazil.

${ }^{2}$ Orthodontist. MSc student in Orthodontics, University Sagrado Coração (USC), Brazil.

${ }^{3} \mathrm{MSc}, \mathrm{PhD}$ candidate in Orofacial Clefts and Related Anomalies, HRAC/USP, Brazil. Orthodontist of the dental division of the HRAC/USP. Professor of Preventive and Interceptive Orthodontics, PROFIS, Brazil.
How to cite this article: Silva Filho OG, Marinho ET, Lauris RCMC. Influence of palatoplasty on occlusion of patients with isolated cleft palate. Dental Press J Orthod. 2012 Mar-Apr;17(2):104-12.

\footnotetext{
Submitted: September 25, 2008 - Revised and accepted: February 17, 2010

" The authors report no commercial, proprietary, or financial interest in the products or companies described in this article.

» Patients displayed in this article previously approved the use of their facial and intraoral photographs.

Contact address: Rita de Cássia Moura Carvalho Lauris

R. Silvio Marchione, 3-20 - Vila Universitária - CEP: 17012-900 - Bauru/SP - Brazil

E-mail: ritalauris@yahoo.com.br
} 


\section{INTRODUCTION}

Derived from a fusion failure of the secondary palatal processes in intrauterine life, the isolated cleft palate is established between the eighth and twelfth week of gestation. The morphological manifestation of cleft palate increases in severity from the uvula towards the alveolar ridge, reaching the incisive foramen when is complete (Fig 1). Isolated cleft palate accounts for $25 \%$ of all cases of cleft lip/palate and is more severe and frequent in females. Data from the archives of the Craniofacial Anomaly Rehabilitation Hospital of University of São Paulo in the city of Bauru, state of São Paulo, Brazil, on 9821 patients with isolated cleft palate confirm the predominance of females (59.63\%) over males (40.37\%). While there is an agreement among authors regarding the multifactorial etiology of this type of cleft, current studies stress the polygenic origin in the palate formation process. ${ }^{23,24}$ The treatment of a cleft palate begins with a primary palatoplasty at 12 months of age. ${ }^{25,28}$ From that point on, non-surgical therapy is then directed mainly towards vocal quality and hearing competence.

In cases of isolated cleft palate, the dentofacial morphology defined by cephalometrics appears to be inherent to the cleft itself and is little affected by palatoplasty. ${ }^{4,5,20,25,26}$ Regardless if a palatoplasty was performed in childhood, the cephalometric dimensions in patients with isolated cleft palate translate into small, well related and proportionally retro-positioned apical bases, along with a clockwise rotation of the mandible, denoting a preponderantly vertical growth. ${ }^{6,8,14} \mathrm{~A}$ study on facial morphology through a clinical analysis of the face confirms the cephalometric diagnosis and the small influence of palatoplasty on the final configuration of the face. ${ }^{28}$ It can be concluded that the facial morphology of patients with isolated cleft palate is inherent to the individual and undergoes little influence from palatoplasty.

With regards to the occlusion, the literature has shown that palatoplasty in isolated cleft palate affects the development of the upper dental arch, with a reduction in width ${ }^{11,15,20,29}$ (Fig 2A) or in width and length ${ }^{3,7,10,12,14,21,22}$ with variations depending on the surgical technique ${ }^{15,20}$ and on number of surgeries performed..$^{10}$ Moreover, the lower arch tends to follow the constriction of the upper arch (Fig 2B). ${ }^{3}$

The dimensions of the alveolar ridge in patients with isolated cleft palate are already reduced before the eruption of the primary teeth, regardless of the extension of the cleft, ${ }^{7}$ and remain reduced throughout the three developmental stages of the occlusion (primary, mixed and permanent dentition). ${ }^{3}$ Quantitative studies involving study models show that the dimensions of the dental arch in operated cleft patients are
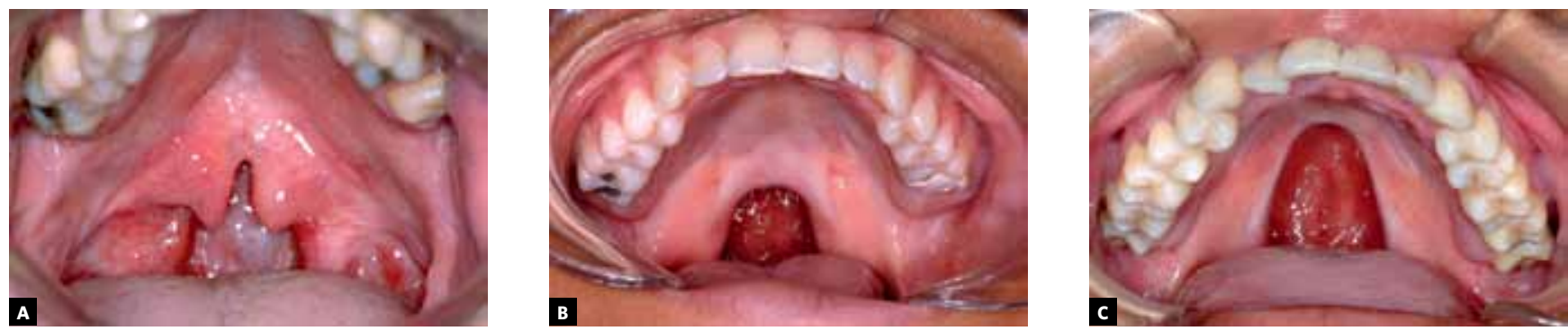

Figure 1 - A) Isolated cleft palate: Bifid uvula, B) Isolated cleft palate: Soft palate, C) Isolated cleft palate: Soft and hard palate.

Figure 2 - A) Crowding in the upper arch in an isolated cleft palate patient. B) Crowding in the lower arch in an isolated cleft palate patient: The lower dental arch tends to accompany the upper arch, demonstrating a reduction in its transverse dimensions.
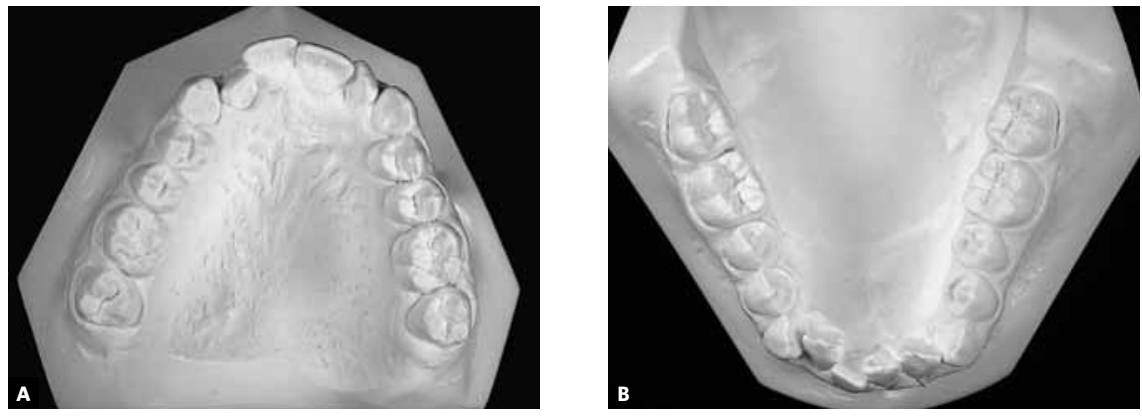
reduced in the primary dentition, ${ }^{12}$ beginning at three years of age and remaining so through $\operatorname{six}^{20}$ or even ten years of age, ${ }^{7}$ regardless of the surgical technique employed. ${ }^{21}$ On the permanent dentition, the palate is narrower throughout its entire length and shallower from the premolars towards the molars..$^{29}$ In patients who did not undergo surgery when newborns, the width of the alveolar ridge is influenced by the width of the cleft, with wider clefts leading to larger dimensions in the alveolar arch. ${ }^{12}$

The literature is unclear regarding the influence of palatoplasty on the width of the upper dental arch. Even though more conservative techniques, that expose the palate less, are reported to have a smaller effect on the width of the upper dental arch, at least in the primary dentition phase, ${ }^{15}$ this is not a widespread opinion. ${ }^{7,14}$ It is also reported that the shape of the upper dental arch may be related to the scar tissue of the palate. ${ }^{13}$

The aim of the present study was to determine the morphological characterization of the occlusion based on the analysis of study models, comparing isolated cleft palate adults that underwent surgery in childhood with those that did not undergo surgery.

\section{MATERIAL AND METHODS}

The sample consisted of 77 patients treated at the Craniofacial Anomaly Rehabilitation Hospital of the University of São Paulo (Brazil) with isolated cleft palate (30 males and 47 females) (Table 1). Mean age was 21 years, ranging from 11 years and two months to 39 years and eight months and all patients were in the permanent dentition. Thirty-nine had not been submitted to palatoplasty, while thirty-eight had been submitted to palatoplasty in childhood at a mean age of 2.2 years by different surgeons employing different surgical techniques.

The inclusion criteria were: Brazilian nationality, absence of syndromes, permanent dentition stage, no prior orthodontic treatment and complete or incomplete isolated cleft palate, whether or not having been submitted to palatoplasty. In the operated cases, the first surgery could not have been done after four years of age. Study models of both dental arches were obtained from the subjects and occlusion analysis was performed by two previously calibrated orthodontists, considering the inter-arch and intraarch relationships.
The following characteristics were analyzed:

\section{» Inter-arch relationship}

- Sagittal relationship: Class I, II or III, according to premolars;

- Transverse relationship: Presence or absence of unilateral/bilateral posterior crossbite;

- Incisor relationship: Increased overbite and overjet, edge-to-edge or anterior open bite/anterior crossbite.

\section{» Intra-arch relationship}

- Presence or absence of anterior and posterior crowding;

- Arch shape: Normal or constricted.

The sample was divided into two groups (39 patients who had not undergone palatoplasty and 38 patients who had undergone surgery in childhood) and were further subdivided into complete cleft and incomplete cleft (Table 1). Due to a small number of non-operated patients with complete cleft, the statistical analysis did not include the extension of the cleft in the non-operated group. Agreement between the two orthodontists in the evaluation of the occlusal characteristics analyzed was determined using the Kappa coefficient ${ }^{2}$ (Table 2).

For comparisons between groups of non-operated patients with isolated cleft palate, operated patients with complete cleft and operated patients with incomplete cleft, the exact chi-square test ${ }^{1}$ was used, with the level of significance set at $5 \%(p<0.05)$. In cases where $\mathrm{p}<0.05$, a comparison was made between the operated groups with complete and incomplete cleft. When the difference between groups was insignificant $(p<0.05)$, the groups with complete and incomplete cleft were

Table 1 - Distribution of patients with isolated cleft palate according to gender and palatoplasty (in percentages)

\begin{tabular}{clccc} 
& & Male & Female & Total \\
\hline \multirow{3}{*}{ Non-operated } & Incomplete & 15 & 18 & 33 \\
\cline { 2 - 4 } & Complete & 2 & 4 & 6 \\
\cline { 2 - 4 } Operated & Total & 17 & 22 & 39 \\
& Incomplete & 12 & 15 & 27 \\
& Complete & 1 & 10 & 11 \\
\cline { 2 - 4 } Overall sample & Total & 13 & 25 & 38 \\
\hline
\end{tabular}


pooled and the exact chi-square test was performed again considering the surgery factor alone. In cases where $\mathrm{p}<0.05$, all groups were compared using exact chi-square tests, with the level of significance defined as $\alpha=0.05 / p=0.017$ (Bonferroni correction). ${ }^{19}$

\section{RESULTS}

The results (Table 2) show a high level of agreement between the two examiners in the occlusal evaluation, demonstrating the reliability of the study model's analysis as a criterion of morphological analysis in orthodontic diagnosis.

Table 3 displays the comparisons between groups. A significant difference was detected in upper alignment/crowding between non-operated and operated patients with complete cleft $(\mathrm{p}<0.001)$. A significant difference in upper arch shape was detected between the operated and non-operated groups $(p=0.0018)$.

\section{DISCUSSION}

Among the 77 patients observed, 5 (6.5\% of the sample) exhibited normal occlusion (Table 4) while the vast majority (93.5\%) exhibited some type of malocclusion. Considering the surgery variable, the prevalence of normal occlusion was $10.25 \%$ among the non-operated patients and $2.7 \%$ among the operated patients. Despite the small sample size, the greater percentage of normal occlusion among the non-operated patients in comparison to those that underwent palatoplasty in childhood suggests that this type of surgery somehow deteriorates the occlusion.

Even tough malocclusion prevailed in the patients, there was not a typical malocclusion for the isolated cleft palate. Malocclusion characteristics were identified in three dimensions, with a predominance of Class II malocclusion as well as upper and lower crowding.

Table 2 - Kappa confidence intervals for agreement between two examiners.

\begin{tabular}{lccc}
\multicolumn{1}{c}{ Variable } & Kappa estimate & \multicolumn{2}{c}{ Confidence interval (95\%) } \\
& & Lower limit & Upper limit \\
Sagittal relationship & 0.98 & 0.95 & 1.00 \\
Transverse relationship & 0.96 & 0.90 & 1.00 \\
Overbite & 0.98 & 0.94 & 1.00 \\
Overjet & 0.94 & 0.88 & 1.00 \\
Upper crowding & 0.96 & 0.89 & 1.00 \\
Upper arch shape & 0.97 & 0.92 & 1.00 \\
Lower crowding & 0.98 & 0.94 & 1.00 \\
Lower arch shape & 0.96 & 0.88 & 1.00 \\
\hline
\end{tabular}

Table 3 - Results of chi-square test in comparison between groups.

\begin{tabular}{|c|c|c|c|c|c|}
\hline \multirow[b]{2}{*}{ Variable } & \multirow[b]{2}{*}{$\mathbf{p}$} & \multicolumn{4}{|c|}{ Comparisons between groups } \\
\hline & & $\begin{array}{l}\text { N-Op X } \\
\text { Op comp }\end{array}$ & $\begin{array}{c}\text { N-Op X } \\
\text { Op inc }\end{array}$ & $\begin{array}{c}\text { Op comp X } \\
\text { Op inc }\end{array}$ & $\begin{array}{c}N-O p X \\
O p\end{array}$ \\
\hline Sagittal relationship & 0.2322 & & & & \\
\hline Transverse relationship & 0.1804 & & & & \\
\hline Overbite & 0.1398 & & & & \\
\hline Overjet & 0.7994 & & & & \\
\hline Upper alignment/crowding * & 0.0002 & $<0.001$ & 0.0220 & 0.0343 & \\
\hline Upper arch shape & 0.0238 & & & 0.5076 & 0.0018 \\
\hline Lower Alignment / Crowding & 0.2891 & & & & \\
\hline Lower arch shape & 0.0629 & & & & \\
\hline
\end{tabular}


Table 4 - Distribution of normal occlusion and malocclusion in sample of 77 patients with isolated cleft palate according to operated and non-operated subgroups (in percentages).

\begin{tabular}{ccc} 
& Normal occlusion & Malocclusion \\
\hline Overall sample & 6.50 & 93.50 \\
Non-operated cleft & 10.25 & 89.75 \\
Operated cleft & 2.70 & 97.30 \\
\hline
\end{tabular}

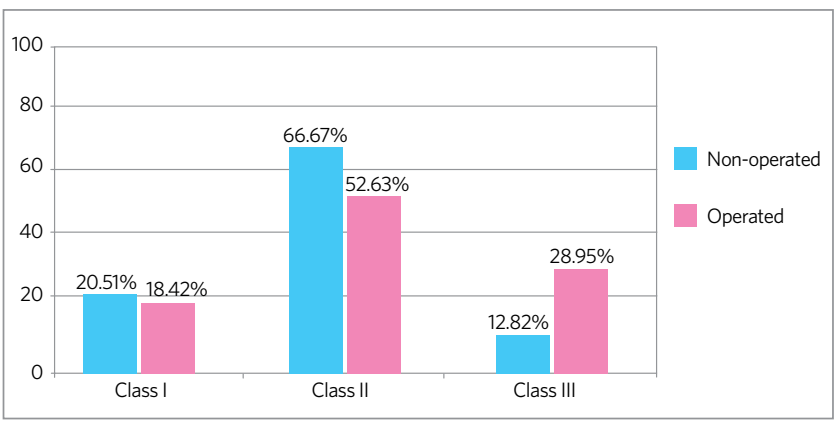

Figure 3 - Percentage distribution of sagittal relationship between dental arches (Class I, Class II and Class III) in sample of 77 patients with isolated cleft palate.

\section{SAGITTAL RELATIONSHIP}

A Class I relationship between the arches occurred in only $20 \%$ of the patients, whereas $80 \%$ exhibited sagittal discrepancies, distributed as $59.74 \%$ with Class II and $20.78 \%$ with Class III. Distribution was similar when considering the operated and nonoperated groups, even though there were more cases of Class III among the operated patients (28.95\%) than in the non-operated patients $(12.82 \%)$. No differences were detected in these groups or in relation to the extension of the cleft (Fig 3).

The larger number of Class II malocclusions may be explained by the condition of the mandible, which exhibits a predominantly vertical growth in cases of cleft as well as morphological, spatial and dimensional abnormalities. ${ }^{6,8,14}$ Cephalometric data on the mandible showed a clockwise rotation, ${ }^{25,28}$ an increased mandibular plane angle, a retrusion in relation to the cranial base $\mathrm{e}^{14}$ and a reduction on the dimensions of the body and ramus. ${ }^{8,27,30}$ The clockwise rotation of the mandible can help to explain the increased incidence of Class II malocclusion in patients with isolated cleft palate.
Class III malocclusion was high (21\%) in relation to what is expected in Caucasian patients with no malformations. The fact that palatoplasty had no significant influence over the sagittal relationship between the arches suggests that the Class III relationship was not determined by this surgery, but rather is a part of the morphological condition of the cleft patient. The data suggest that cleft palate has a negative influence over the sagittal relationship between the maxilla and mandible (Fig 3).

\section{TRANSVERSE RELATIONSHIP}

The literature shows that the transverse dimensions of the upper arch are small in cases of isolated cleft palate, regardless of the extension of the cleft or surgical technique employed. Studies that quantitatively assess the transverse dimension of the dental arches report a reduction of this dimension on the upper arch during the three developmental stages of the occlusion: Primary, ${ }^{3,7,11,12,14,20,21,22}$ mixed $^{3,7,13,14}$ and permanent., ${ }^{3,29}$ The literature also suggests that the lower arch tends to follow the upper arch, diminishing its transverse dimensions during the primary dentition, ${ }^{3,12,20,22}$ on the other developmental stages of the occlusion $^{3}$ and on the permanent dentition. . $^{3,11}$

On the other hand, posteroanterior cephalometries show that an isolated cleft palate causes a morphological craniofacial condition with increased transverse dimensions, as shown by a greater lateral inclination of the pterygoid processes of the sphenoid bone, by an increase of the distance between the hamular processes, and by a greater width in the region of the tuberosity. Pressure from palatoplasty does not affect the width of the basal portion of the maxilla. ${ }^{4}$ Frontal cephalometries also reveal that the surgical technique employed does not affect facial or occlusal behavior. ${ }^{9}$

Based on the data obtained from PA cephalometries, the reduction in the transverse dimension in cases of isolated cleft palate seems to be linked to the upper dental arch and alveolus. This data may also infer that the mid-face does not exhibit the same transverse behavior as that of the dental arch and alveolus.

If the transverse reduction were only present in the operated cases, it could be attributed to the scar tissue of the palatoplasty. Laboratory studies accept the theory that post-palatoplasty scar tissue reduces 
the width of the upper dental arch. ${ }^{16,17}$ From the clinical standpoint, however, it is difficult to determine the degree of this interference, even though the shape of the upper arch has been found to be related to the intensity of the scar tissue in the palate. ${ }^{13}$ It is likely that the influence of palatoplasty on the upper dental arch is individual.

In the present study, transverse morphological condition was assessed considering the inter-arch relationship, evaluating the presence of posterior crossbite, the intra-arch relationship and the morphology of the upper and lower dental arches. The incidence of posterior crossbite was $23.38 \%$, regardless of the palatoplasty and the severity of the cleft (Table $5)$. The incidence of posterior crossbite was lower than that reported in the literature for five-year-old children with isolated cleft palate operated between 18 and 24 months of age. ${ }^{12}$ In children, the incidence of posterior crossbite was $14.1 \%$, and $19.2 \%$ when combined with anterior crossbite, totaling $33.3 \%$. These epidemiological data suggest that the cleft itself in combination with palatoplasty influences the transverse behavior of the occlusion.

Table 5 - Distribution of posterior crossbite (PCB) on 77 patients with isolated cleft palate according to operated and non-operated subgroups and extension of cleft (in percentages).

\begin{tabular}{cccc} 
& & Normal & PCB \\
\hline \multirow{3}{*}{ Non-operated } & Incomplete & 72.73 & 27.27 \\
& Complete & 66.67 & 33.33 \\
& Total & 71.79 & 28.21 \\
& Incomplete & 81.48 & 18.52 \\
Operated & Complete & 81.82 & 18.18 \\
& Total & 81.58 & 18.42 \\
\hline \multirow{2}{*}{ Overall sample } & & 76.62 & 23.38 \\
\hline
\end{tabular}

Table 6 - Distribution of upper arch shape in sample of 77 patients with isolated cleft palate according to operated and non-operated subgroups and extension of cleft (in percentages).

\begin{tabular}{cccc} 
& & Normal & Constriction \\
\hline \multirow{3}{*}{ Non-operated } & Incomplete & 75.76 & 24.24 \\
& Complete & 66.67 & 33.33 \\
& Total & 74.36 & 25.64 \\
& Incomplete & 48.15 & 51.85 \\
Operated & Complete & 36.36 & 63.64 \\
& Total & 44.74 & 55.26 \\
Overall sample & & 59.74 & 40.26 \\
\hline
\end{tabular}

The results on transverse dimension exclude palatoplasty as an interfering variable on the interarch relationship. The analysis of that relationship revealed that one-third $(76.62 \%)$ of the sample had a normal transverse relationship, whereas $23.38 \%$ of the overall sample had some type of posterior crossbite (28.21\% of the non-operated group and $18.42 \%$ of the operated group). If palatoplasty can reduce the width of the upper arch, a greater prevalence of posterior crossbite was expected among the operated patients. The results, on the other hand, reveal that the transverse inter-arch relationship was not influenced by either palatoplasty or by the extension of the cleft.

The incidence of posterior crossbite was low, considering the morphology of the upper dental arch. Approximately $40 \%$ of the patients exhibited a constriction of the upper arch (Table 6). The percentage of constriction in the upper arch was larger than that of the lower arch (Table 7). However, this percentage was larger than that of posterior crossbite in this same group (18.42\%). There are two important considerations to be discussed: The incidence of Class II (approximately 60\%) and the incidence of lower arch constriction (approximately 21\%). It can be concluded that the Class II relationship and the constriction in the lower arch contribute toward masking the constriction of the upper arch when diagnosis is made considering the inter-arch relationship.

In regards to the intra-arch relationship, the percentage of constriction in the upper arch was significantly larger in the operated group than in the non-operated group. The same occurred in the lower arch, but without significance. This finding suggests the influence of palatoplasty on the upper arch shape, which reflects on the lower arch's tendency in

Table 7 - Distribution of lower arch shape in 77 patients with isolated cleft palate according to operated and non-operated subgroups and extension of cleft (in percentages).

\begin{tabular}{cccc} 
& & Normal & Constriction \\
\hline \multirow{2}{*}{ Non-operated } & Incomplete & 90.91 & 9.09 \\
& Complete & 83.33 & 16.67 \\
& Total & 89.74 & 10.26 \\
& Incomplete & 70.37 & 29.63 \\
Operated & Complete & 63.63 & 36.37 \\
& Total & 68.42 & 31.58 \\
Overall sample & & 79.22 & 20.78 \\
\hline
\end{tabular}


adjusting itself to the upper arch in the transverse dimension. A higher percentage of constriction was noted in both arches among the patients with complete cleft in comparison to those with incomplete cleft, but this difference was insignificant.

An important aspect of malocclusion is that the transverse problem does not compromise the prognosis of treatment - at least until the end of adolescence. It is possible to correct constriction of the upper arch in the primary, mixed and permanent dentition. The transverse dimension does not determine the prognosis of treatment.

\section{CROWDING IN UPPER AND LOWER ARCHES}

Crowding occurred in nearly $50 \%$ of the sample, more precisely in $46.75 \%$ in the upper arch and $51.95 \%$ in the lower arch. A part of the crowding was related to the constriction of both arches. Approximately $40 \%$ and $21 \%$ of the patients with isolated cleft palate had a diagnosis of constriction in the upper and lower arches, respectively.

On the upper arch, when the surgery factor is considered, crowding occurred in $65.79 \%$ of the operated patients and $28.21 \%$ of the non-operated patients. This significant difference suggests the influence of palatoplasty on the shape of the upper arch, because $55.26 \%$ of the operated patients exhibited constricted arches in comparison to the $25.64 \%$ of non-operated patients, also with a significant difference (Table 4).

In regards to the lower arch, no significant difference was found in crowding between groups, but once again there was a tendency towards larger crowding on

Table 8 - Distribution of alignment/crowding in upper arch in $77 \mathrm{pa}$ tients with isolated cleft palate according to operated and non-operated subgroups and extension of cleft (in percentages).

\begin{tabular}{cccc}
\hline \multirow{3}{*}{ Non-operated } & Alignment & Crowding \\
& Incomplete & 75.76 & 24.24 \\
& Complete & 50.00 & 50.00 \\
& Total & 71.79 & 28.21 \\
& Incomplete & 44.44 & 55.56 \\
Operated & Complete & 9.09 & 90.91 \\
& Total & 34.21 & 65.79 \\
Overall sample & & 53.25 & 46.75 \\
\hline
\end{tabular}

the operated group (63.16\%) in comparison to the nonoperated group (41.03\%). The same occurred when the lower arch shape is analyzed, with a larger number of arch constriction in the operated group (31.58\%) compared to the non-operated group (10.26\%).

Despite the lack of significance, more cases of crowding were found on the operated patients with complete cleft in both arches $(90.91 \%$ for the upper arch and $72.73 \%$ for the lower arch), implying that the extension of the cleft is also involved on the shape of the dental arches after the surgical closure of the palate (Tables 8 and 9).

\section{OVERBITE AND OVERJET}

Approximately $20 \%$ of the patients exhibited increased overbite and overjet. It is likely that these are a part of the Class II malocclusion, while $32 \%$ of the patients exhibited an overjet ranging from edge-toedge $(17.72 \%)$ to anterior cross-bite (14.28\%). When the surgery factor was considered, there was a greater sagittal discrepancy of the incisors on the patients that underwent palatoplasty. This fact is based on the reduction of the overjet and increased incidence of anterior crossbite (ACB) on the operated group in comparison to the non-operated group (Table 10).

The amount of anterior open bites (AOB) was $15 \%$, by adding it to the edge-to-edge (EtE) relationship, vertical problems on the incisors affected $36.36 \%$ of the patients. The treatment prognosis is poor, mainly due to the tendency towards vertical mandibular growth in patients with isolated cleft palate. In all the groups studied, normal incisor relationship occurred

Table 9 - Distribution of alignment/crowding in lower arch in $77 \mathrm{pa}-$ tients with isolated cleft palate according to operated and non-operated subgroups and extension of cleft (in percentages).

\begin{tabular}{cccc} 
& & Alignment & Crowding \\
\hline \multirow{3}{*}{ Non-operated } & Incomplete & 60.60 & 39.40 \\
& Complete & 50.00 & 50.00 \\
& Total & 58.97 & 41.03 \\
& Incomplete & 40.74 & 59.26 \\
Operated & Complete & 27.27 & 72.73 \\
& Total & 36.84 & 63.16 \\
Overall sample & & 48.05 & 51.95 \\
\hline
\end{tabular}


Table 10 - Distribution of horizontal overjet in sample of 77 patients with isolated cleft palate according to operated and non-operated subgroups and extension of cleft (in percentages).

\begin{tabular}{ccccc}
\hline \multirow{3}{*}{ Non-operated } & Increased & Normal & EtE+ACB \\
\cline { 3 - 5 } & Incomplete & 21.21 & 54.55 & 24.24 \\
& Complete & 50.00 & 16.67 & 33.33 \\
\cline { 3 - 5 } Operated & Total & 25.64 & 48.72 & 25.64 \\
& Incomplete & 14.81 & 48.15 & 37.04 \\
& Complete & 9.09 & 45.46 & 45.45 \\
Overall sample & Total & 13.16 & 47.37 & 39.47 \\
\hline \multirow{2}{*}{} & & 19.48 & 48.05 & 32.47 \\
\hline
\end{tabular}

less in the operated group compared to the non-operated group, as reflected in the greater number of cases of increased overbite and anterior open bite in the former group (Table 11). The statistical relevance was null regarding the effect of the surgery factor and extension of the cleft on the incisor relationship.

\section{CONCLUSIONS}

On isolated cleft palate, malocclusions were found to have different manifestations, not only regarding
Table 11 - Distribution of vertical overjet in sample of 77 patients with isolated cleft palate according to operated and non-operated subgroups and extension of cleft (in percentages).

\begin{tabular}{ccccc}
\hline \multirow{2}{*}{ Non-operated } & Increased & Normal & EtE+AOB \\
\cline { 3 - 5 } & Incomplete & 9.09 & 60.61 & 30.30 \\
\cline { 3 - 5 } Operated & Complete & 16.67 & 33.33 & 50.00 \\
& Total & 10.26 & 56.41 & 33.33 \\
& Incomplete & 33.33 & 29.63 & 37.04 \\
Overall sample & Complete & 9.09 & 45.46 & 45.45 \\
\hline \multirow{2}{*}{ Total } & 26.32 & 34.21 & 39.47 \\
\hline
\end{tabular}

inter-arch relationships (Class I, Class II and Class III) but also incisor relationships (overbite and overjet). The predominant morphological characteristics in these patients included Class II malocclusion, constriction in the upper arch and crowding in both arches. The influence of palatoplasty was restricted to constriction and crowding in the upper arch. The extension of the cleft had influence only over the crowding of the upper arch, which occurred more in patients with complete cleft. 


\section{REFERENCES}

1. Agresti A. A survey of exact inference for contingency tables. Stat Sci. 1992;7(1):131-77.

2. Agresti A. Categorical Data Analysis. $2^{\text {nd }}$ ed. New York: Wiley; 2002.

3. Athanasiou AE, Mazaheri M, Zarrinnia K. Longitudinal study of the dental arch dimensions in hard and soft palate clefts. J Pedod. 1987;12(1):35-47.

4. Athanasiou AE, Moyers RE, Mazaheri M, Toutountzakis N. Frontal cephalometric evaluation of transverse dentofacial morphology and growth of children with isolated cleft palate. J Craniomaxillofac Surg. 1991;19(6):249-53.

5. Bishara SE. Cephalometric evaluation of facial growth in operated and non-operated individuals with isolated clefts of the palate. Cleft Palate J. 1073;10(3):239-46.

6. Bishara SE. The influence of palatoplasty and cleft length on facial development. Cleft Palate J. 1973;10(4):390-8

7. Friede $\mathrm{H}$, Enocson L, Möller M, Owman-Moll P. Maxillary dental arch and occlusion in patients with repaired clefts of the secondary palate. Influence of push back palatal surgery. Scand J Plast Reconstr Surg Hand Surg. 2000;34(3):213-8.

8. Fujita S, Suzuki A, Nakamura N, Sasaguri M, Kubota Y, Ohishi M. Retrospective evaluation of craniofacial growth of Japanese children with isolated cleft palate: from palatoplasty to adolescence. Cleft Palate Craniofac J. 2005;42(6):625-32.

9. Heliövaara A. One-stage closure of isolated cleft palate with the Veau-WardillKilner $V$ to $Y$ pushback procedure or the Cronin modification. IV. Cephalometric comparison of transverse dentofacial morphology. Eur J Orthod. 1994;16(1):63-9.

10. Heliövaara A, Pere A, Ranta R. One-stage closure of isolated cleft palate with the Veau-Wardill-Kilner $V$ to $Y$ pushback procedure or the Cronin modification. II. Height, weight and comparison of dental arches. Scand J Plast Reconstr Surg Hand Surg. 1994;28(1):55-62.

11. Heliövaara A, Rautio J. Dental arches in six-year-old children with operated and unoperated submucous cleft palate and isolated cleft palate. Acta Odontol Scand. 2005;63(2):123-6

12. Hellquist $R$, Pontén $B$, Skoog $T$. The influence of cleft length and palatoplasty on the dental arch and the deciduous occlusion in cases of clefts of the secondary palate. Scand J Plast Reconstr Surg. 1978;12(1):45-54.

13. Ishikawa H, Nakamura S, Misaki K, Kudoh M, Fukuda H, Yoshida S. Scar tissue distribution on palates and its relation to maxillary dental arch form. Cleft Palate Craniofac J. 1998;35(4):313-9.

14. Jonsson G, Thilander B. Occlusion, arch dimensions, and craniofacial morphology after palatal surgery in a group of children with clefts in the secondary palate. Am J Orthod. 1979;76(3):243-55.

15. Karsten A, Larson M, Larson O. Dental occlusion after Veau-Wardill-Kilner versus minimal incision technique repair of isolated clefts of the hard and soft palate. Cleft Palate Craniofac J. 2003:40(5):504-10.
16. Kremenak CR, Huffman WC, Olin WH. Growth of maxillae in dogs after palatal surgery: I. Cleft Palate J. 1967;4(1):6-17.

17. Kremenak CR Jr, Huffman WC, Olin WH. Maxillary growth inhibition by mucoperiosteal denudation of palatal shelf bone in non-cleft beagles. Cleft Palate J. 1970;7(4):817-25.

18. Landis JR, Koch GG. The measurement of observer agreement for categorical data. Biometrics. 1977;33(1):159-74.

19. Neter J, Kutner M, Wasserman W, Nachtsheim C, Neter J. Applied linear statistical models. $4^{\text {th }}$ ed. Chicago: Irwin; 1996.

20. Nyström M, Ranta R. Effect of timing and method of cleft palate repair on dental arches at the age of three years. Cleft Palate J. 1990;27(4):349-52; discussion 352-3.

21. Nyström M, Ranta R. Effect of timing and method of closure of isolated cleft palate on development of dental arches from 3 to 6 years of age. Eur J Orthod. 1994:16(5):377-83

22. Nyström M, Ranta R, Kataja M. Sizes of dental arches and general body growth up to 6 years of age in children with isolated cleft palate. Scand J Dent Res. 1992;100(2):123-9.

23. Qiu M, Bulfone A, Ghattas I, Meneses JJ, Christensen L, Sharpe PT, et al. Role of the D1x homeobox genes in proximodistal patterning of the branchial arches: mutations of D1x-1, D1x-2, and D1x-1 and -2 alter morphogenesis of proximal skeletal and soft tissue structures derived from the first and second arches. Dev Biol. 1997;185(2):165-84.

24. Satokata I, Maas R. Msx 1 deficient mice exhibit cleft palate and abnormalities of craniofacial and tooth development. Nat Genet. 1994;6(4):348-56.

25. Silva Filho OG, Cavassan AO, Normando ADC. Influência da palatoplastia no padrão facial de pacientes portadores de fissura pós-forame incisivo. Rev Bras Cir. 1989;79(6):315-22

26. Silva Filho OG, Normando AD, Capelozza Filho L. Mandibular morphology and spatial position in patients with clefts: intrinsic or iatrogenic? Cleft Palate Craniofac J. 1992;29(4):369-75

27. Silva Filho OG, Normando ADC, Capelozza Filho L. Mandibular growth in patients with cleft lip and/or cleft palate - the influence of cleft type. Am J Orthod Dentofacial Orthop. 1993;104(3):269-75.

28. Silva Filho OG, Rosa LAA, Lauris RCMC. Influence of isolated cleft palate and palatoplasty on the face. J Appl Oral Sci. 2007;15(3):199-208.

29. Smahel Z, Trefný P, Formánek P, Müllerová Z, Peterka M. Three-dimensional morphology of the palate in subjects with isolated cleft palate at the stage of permanent dentition. Cleft Palate Craniofac J. 2003;40(6):577-84.

30. Smahel Z. Variations in craniofacial morphology with severity of isolated cleft palate. Cleft Palate J. 1984;21(3):140-58 